\title{
15 Von der Kunst des Impliziten zur Last des Verborgenen bei Nathalie Sarraute
}

\subsection{Sarraute und die Entdeckung der ,sous-conversation`}

Nathalie Sarraute beginnt ihre schriftstellerische Karriere in den dreißiger Jahren. Unmittelbar im Anschluss an die Romane Prousts verfasst sie ab 1932 nach und nach einzelne Erzählstücke, die 1939 von der Öffentlichkeit weitgehend unbemerkt unter dem Titel Tropismes als Erstlingswerk veröffentlicht werden. Erst mit der Neuedition aus dem Jahr 1957 erhalten die Erzählstücke der Tropismes den Status eines Gründungswerks des ,nouveau roman' und stoßen auf die Aufmerksamkeit einer größeren Öffentlichkeit. Lange Zeit nach der Abfassung dieser kurzen, auf den ersten Blick impressionistischen Erzählungen stellt Sarraute Überlegungen zur Geschichte des Romans und zur literarhistorischen Situierung ihrer Erzählungen an, die sie mit dem bekannten Band L'Ėre du soupçon (1955) präsentiert. Die Tropismes wie die literaturtheoretischen Überlegungen umkreisen Fragen der Konversation, verstanden im ursprünglichen Sinn des Begriffs, als Umgang der Menschen miteinander. Sarraute macht auf eine besondere, indirekte Dimension der Kommunikation aufmerksam, die sowohl in den Traktaten zur Konversation als auch in den literarischen Reflexionen zur Rolle und Bedeutung der Konversation in Frankreich bis dahin weitgehend unterbelichtet geblieben ist: Ihr gesamtes erzählerisches Werk dreht sich um die Sichtbar- bzw. Spürbarmachung unterschwelliger Spannungen, die Kommunikationen wesentlich steuern. Dabei handelt es sich um spontan-emotionale, psychische Reaktionen auf Aussagen und Verhaltensweisen beteiligter Personen, die an der Kommunikation beteiligt sind. Für Sarraute liegen solche Spannungen jenseits einer konkreten sozialen und historischen Verortung und sind als anthropologische Gegebenheiten anzusehen. Sie hat es also ihrem Selbstverständnis zufolge nicht auf die speziellen kulturellen Besonderheiten der indirekten Kommunikation in Frankreich abgesehen, sondern auf grundlegende allgemeinmenschliche Vorgänge. Gleichwohl ist es nicht verwunderlich, dass gerade eine französische Autorin sich mit jenen psychischen Spannungen befasst, die eine besondere Dimension der indirekten Kommunikation darstellen. Die von Sarraute als ,Tropismen bezeichneten Reibungen sind in der Regel Ausdruck vertikaler Kommunikationsverhältnisse, wie sie besonders typisch für die französische Kommunikation sind. Sie spielen sich meistens zwischen unter- und übergeordneten Personen ab. Sie sind getragen von einer augustinisch geprägten ,negativen Anthropologie‘, die das moralistische Denken im Zentralstaat des französischen 17. Jahrhunderts geprägt hatte. 
Auf diese Weise reflektiert Sarraute zwei der wichtigsten Dimensionen des französischen Verständnisses von Kommunikation: Zum einen die vertikale Dimension, die von Machtsituationen bestimmt ist. Die ,bienséances‘ werden bei ihr als Unterdrückungsmechanismen der Sprache und Kommunikation angesehen, gegen die die betroffenen Personen mit Irritationen reagieren und gelegentlichen Ausbrüchen revoltieren. Zum anderen wird der Sprache und der Kommunikation jedwede Leichtigkeit genommen, wie sie seit dem 17. Jahrhundert als Charakteristikum insbesondere der französischen Konversation angesehen wird. Die so leicht und spielerisch daherkommende Sprache wird als Sprache der Unterdrückung entlarvt.

Tropismen bezeichnen in der Botanik Richtungsänderungen des Pflanzenwachstums unter dem Einfluss von Licht und Schwerkraft. Sarraute überträgt diese Bezeichnung auf den Bereich des zwischenmenschlichen Umgangs, der für sie ganz wesentlich von nahezu unsichtbaren Reibungen der Kommunikationspartner anlässlich bestimmter verbaler oder nonverbaler Äußerungen geprägt ist. Die daraus resultierenden Abwehr- bzw. Unmutsbewegungen, eher selten belustigte oder gar freudige Regungen, sind das Thema des Romanwerks von Sarraute von ihren Anfängen, den Tropismes, bis hin zu ihrem letzten Roman Ouvrez! (1997). In der „Préface“ der Aufsatzsammlung L’Ėre du soupçon definiert Sarraute die Friktionen, die sie zuvor als ,tropismes“ bezeichnet hatte:

Ce sont des mouvements indéfinissables, qui glissent très rapidement aux limites de notre conscience; ils sont à l'origine de nos gestes, de nos paroles, des sentiments que nous manifestons, que nous croyons éprouver et qu'il est possible de définir. ${ }^{1}$

Ihr geht es nicht um eine Beschreibung herausragender Konversationen, wie sie in der Literatur von Mme de La Fayette bis hin zu Proust Gegenstand der literarischen Reflexionen waren, sondern darum, wie in den banalsten Alltagsunterhaltungen intensive Gefühlsregungen (,des sensations souvent très intenses“, S. 1554) unvermittelt zu Tage treten, die die Gesprächspartner oftmals selbst gar nicht wahrnehmen, obgleich sie durchaus größere innere Dramen hervorrufen können:

1 „Préface“, in: Nathalie Sarraute, L’Ėre du soupçon, in: N.S., Euvres complètes, hrsg. von Jean-Yves Tardié, Vivianne Forrestier, Ann Jefferson, Valérie Minogue und Arnaud Rykner (Bibliothèque de la Pléiade. 132), Paris, Gallimard, 1996, S. 1553-1556, hier: S. 1553. Im Folgenden nach dieser Ausgabe im laufenden Text zitiert. Einen Überblick über das Werk von Sarraute gibt Brigitta Coenen-Mennemeier, Nouveau roman (Sammlung Metzler. 296), Stuttgart/Weimar, Metzler, 1996. 
Leur déploiement constitue de véritables drames qui se dissimulent derrière les conversations les plus banales, les gestes les plus quotidiens. Ils débouchent à tout moment sur ces apparences qui à la fois les masquent et les révèlent. (Ebd.)

Der Ausgangspunkt von Sarrautes Überlegungen ist die Frage, was der moderne Romancier des 20. Jahrhunderts zur Erzählliteratur beitragen kann. Wie zahlreiche andere Autoren der Zeit auch beobachtet sie, dass der Roman der Gegenwart mehr und mehr Dialoge wiedergibt. Die Romane werden zu,romans conversants' : „[...] le centre de gravité du roman se déplace: le dialogue y occupe une place chaque jour plus grande. " ${ }^{2}$ Immer weniger werden neue Erzählsituationen generiert und Charaktere geschaffen mit dem Ziel, die Sitten einer Epoche widerzuspiegeln, sondern die Autoren interessieren sich zunehmend für die psychischen Gegebenheiten der zwischenmenschlichen Kommunikation und eröffnen dem Roman dadurch den Gegenstand einer neuen Psychologie:

[...] les modernes ont transporté ailleurs l'intérêt essentiel du roman [...] Il ne se trouve plus pour eux dans le dénombrement des situations et des caractères ou dans la peinture des mœurs, mais dans la mise au jour d'une matière psychologique nouvelle. (S. 1593)

Sarraute knüpft folgerichtig vor allem an Proust an, der wie kein Autor vor ihm die Konversationen seiner Zeitgenossen mit einer unnachahmlichen Akribie und Ausführlichkeit (,excessive minutie“, S. 1595) beschrieben hat. Er ist für sie eine Art Karthograph, der die Konversationen aus der Flugperspektive verzeichnet („le cartographe qui étudie une région en la survolant“, S. 1603). Mit André Gide kritisiert Sarraute, dass die Recherche die Konversationen bestenfalls analysiert („d'avoir fait de l'analyse“, S. 1595) und dass Proust seine Protagonisten aus einer großen Distanz beobachtet habe (,il les a observés d'une grande distance“, ebd.), wodurch sie im grellen Licht der Analyse zu quasi erstarrten Analyseobjekten („carcasses vides“, S. 1590) geworden sind, mit denen er wie mit Puppen spiele:

Ces hommes qu'il voudrait tant connaître et faire connaître, quand il essaie de les montrer se mouvant dans la lumière aveuglante du grand jour, lui semblent n'être que de belles poupées, destinées à amuser les enfants. (Ebd.)

Für Sarraute geht bei Proust gerade das für sie Interessante verloren: jene „substance vivante“, jene „matière trouble et grouillante“ (ebd.), die der Autor unter dem Stichwort des ,moi profond“ hinter den Masken der Personen in der

2 „Conversation et sous-conversation”, in: N. S., L'Ėre du soupçon (= Euvres complètes), S. 15871607, hier: S. 1591. Die Stellenangaben im laufenden Text beziehen sich auf diese Ausgabe. 
Kommunikation eigentlich zum Vorschein bringen wollte und die bei ihm stattdessen weit von der Gegenwart entfernt in den Erinnerungen gleichsam eingefroren sind (,comme figés dans le souvenir“, S. 1595). Proust habe seine Leser niemals jene „sensations“ bzw. „impulsions“ präsentisch erfahren lassen, die sich beim Erzähler Marcel im Zuge seiner ,mémoires involontaires‘ einstellen:

Il a rarement - pour ne pas dire jamais - essayé de les revivre et de les faire revivre au lecteur dans le présent, tandis qu'ils se forment et à mesure qu'il se développent comme autant de drames minuscules ayant chacun ses péripéties, son mystère et son imprévisible dénouement. (Ebd.)

Gegenstand ihres programmatischen Beitrags „L'Ère du soupçon“ (1950) aus der gleichnamigen Schrift sind die unterschwelligen Regungen, die sich bei jeder Konversation einstellen. Unter der Maske vertrauter Worte und Gesten, die wir an der Oberfläche bei einer Person wahrnehmen, verbergen sich ungewöhnliche, zumeist heftige, sich oftmals gewaltsam äußernde Widerständigkeiten („,actions souterraines“, ebd.):

Quelque chose d'insolite, de violent, se cachait sous ces apparences familières. Tous les gestes du personnage en retraçaient quelque aspect; le plus insignifiant bibelot en faisait miroiter une facette. C'était cela qu'il s'agissait de mettre au jour, d'explorer jusqu'à ses extrêmes limites, de fouiller dans tous ses replis [...] (S. 1579 f.)

Diese inneren Dramen, die sich im Einzelnen abspielen, sind Begleitaktionen jedweder Kommunikation. Sie benötigen Gesprächspartner oder imaginäre Gesprächspartner, sobald der Betroffene Selbstgespräche führt. Und der Gesprächspartner kann je nach Situation die Bedrohung darstellen, die jene lebhaften und überaus geschmeidigen Reaktionen auslöst; er kann aber auch das Opfer der Reaktionen auf einzelne Worte und Gesten werden, welche kaum fassbar sind, weil sie einerseits zu Entäußerungen drängen, sich andrerseits jedoch sofort wieder in die Dunkelheit des Verdrängten zurückziehen:

Il [= le partenaire] est la menace, le danger réel et aussi la proie qui développe leur vivacité et leur souplesse; l'élément mystérieux dont les réactions imprévisibles, en les faisant repartir à tout instant et se développer vers une fin inconnue, accentuent leur caractère dramatique. Mais, en même temps qu'afin de toucher ce partenaire, ils montent de nos recoins obscurs vers la lumière du jour, une crainte les refoule vers l'ombre. (S. 1596)

In der moralistischen Reflexion des 17. Jahrhunderts hatte der Duc de La Rochefoucauld anders als der zeitgleiche Rationalismus der Cartesianer solche zwischenmenschlichen Friktionen der Kommunikation beschrieben und sie auf den ungreifbaren ,amour-propre' des Einzelnen zurückgeführt: 
L'amour-propre est l'amour de soi-même, et de toutes choses pour soi; il rend les hommes idolâtres d'eux-mêmes, et les rendrait les tyrans des autres si la fortune leur en donnait les moyens; il ne se repose jamais hors de soi, et ne s'arrête dans les sujets étrangers que comme les abeilles sur les fleurs, pour en tirer ce qui lui est propre. Rien n'est si impétueux que ses désirs, rien de si caché que ses desseins, rien de si habile que ses conduites; ses souplesses ne se peuvent représenter, ses transformations passent celles des métamorphoses, et ses raffinements ceux de la chimie. ${ }^{3}$

Die menschliche Kommunikation steht für La Rochefoucald stets im Zeichen heftiger und lang andauernder Erregungen (,grande et longue agitation“4), die im Hin- und Herfluss ihrer fortwährenden Bewegungen (,le flux et reflux de ses vagues continuelles [...] de ses éternels mouvements“5) für den Einzelnen selbst undurchsichtig bleiben. Die symbolistische Sprachkritik des späten 19. Jahrhunderts ist aus der Perspektive einer kritischen Sprachreflexion zu ganz ähnlichen Erwägungen gelangt. Sie hat gezeigt, das die Begehren, die La Rochefoucauld unter dem Stichwort des ,amour-propre‘ verzeichnet hat, sprachlich kaum adäquat vermittelt werden können. So stellt sich angesichts der sprachlichen Möglichkeiten ein Gefühl der Entfremdung ein. Die Feststellung, dass der Mensch keine vollständige Verfügungsgewalt über die Sprache hat, wird auf vielfältige Weise insbesondere bei Stéphane Mallarmé oder Arthur Rimbaud reflektiert. Diese Überlegungen sind ein unmittelbarer Anknüpfungspunkt Nathalie Sarrautes ${ }^{6}$. Auch sie konstatiert, dass die Worte sich der Beherrschbarkeit ihrer Sprecher entziehen. Sie können die inneren Regungen zum Ausdruck bringen, führen aber zugleich ein Eigenleben, so dass sie die Spannungen oftmals ungewollt auslösen, hervorbringen und ebenso ungewollt auch wieder zum Verschwinden bringen:

[...] nous avons à notre disposition les paroles. Les paroles possèdent les qualités nécessaires pour capter, protéger et porter au-dehors ces mouvements souterrains à la fois impatients et craintifs.

Elles ont pour elles leur souplesse, leur liberté, la richesse chatoyante de leurs nuances, leur transparence ou leur opacité.

Leur flot rapide, abondant, miroitant et mouvant permet aux plus imprudentes d'entre elles de glisser, de se laisser entraîner et de disparaître au plus léger signe de danger. (S. 1597)

3 Maximes et réflexions diverses, hrsg. von Jacques Truchet, Paris, Garnier Flammarion, 1977, S. 91 (Maxime supprimée 1).

4 S. 93.

5 Ebd.

6 Vgl. dazu Godo, Une Histoire: „La foi dans les capacités du langage à transmettre une vérité se dérobe et cède la place à l'ère du soupçon, selon la formule stendhalienne remise à la mode par Nathalie Sarraute.“ (S. 323) 
Worte sind für sie wie Waffen, die regelrechte „Verbrechen“ auslösen, die bei den Gesprächspartnern Reaktionen von „unangenehmen Reizungen“ bis hin zu „Verbrennungen“ auslösen können:

Aussi, pourvu qu'elles présentent une apparence à peu près anodine et banale, elles peuvent être et elles sont souvent en effet, sans que personne y trouve à redire, sans que la victime elle-même ose clairement se l'avouer, l'arme quotidienne, insidieuse et très efficace, d'innombrables petits crimes.

Car rien n'égale la vitesse avec laquelle elles touchent l'interlocuteur au moment où il est le moins sur ses gardes, ne lui donnant souvent qu'une sensation de chatouillement désagréable ou de légère brûlure [...] (Ebd.7)

Für den zeitgenössischen Roman - und darum geht es ihr in erster Linie in "Conversation et sous-conversation" - bedeutet dies, die "drames souterrains“ nicht länger aus der Draufsicht von oben, wie bei Proust, ins Bild zu setzen, sondern sie für den Leser allzeit gegenwärtig erlebbar zu machen:

Il est donc permis de rêver - sans se dissimuler tout ce qui sépare ce rêve de sa réalisation d'une technique qui parviendrait à plonger le lecteur dans le flot de ces drames souterrains que Proust n'a eu le temps que de survoler et dont il n'a observé et reproduit que les grandes lignes immobiles: une technique qui donnerait au lecteur l'illusion de refaire lui-même ces actions avec une conscience plus lucide, avec plus d'ordre, de netteté et de force qu'il ne peut le faire dans la vie, sans qu'elles perdent cette part d'indétermination, cette opacité et ce mystère qu'ont toujours ses actions pour celui qui les vit.

Le dialogue, qui ne serait pas autre chose que l'aboutissement ou parfois une des phases de ces drames se délivrerait alors tout naturellement des conventions et des contraintes que rendaient indispensables les méthodes du roman traditionnel. C'est insensiblement, par un changement de rythme ou de forme, qui épouserait en l'accentuant sa propre sensation, que le lecteur reconnaîtrait que l'action est passée du dedans au-dehors. (S. 1604)

In einem guten Theaterstück können die Schauspieler nicht nur durch den Text des Stücks sondern insbesondere durch die Intonation des gesprochenen Wortes Irritationen hervorrufen, die für den Zuschauer durch entsprechende Reaktionen der angesprochenen Person unmittelbar erfahrbar werden ${ }^{8}$. Dies gelte es auch für

7 Zur Frage einer der Alltagssprache vorausgehenden ursprünglichen Sprache der Kindheit bei Sarraute vgl. Layla Raïd, „Sous les mots, l'enfance. Sarraute et la philosophie du langage ordinaire”, A contrario Bd. 25/2017, H. 2, S. 93-116.

8 „Car le dialogue de théâtre, qui se passe de tuteurs, où l'auteur ne fait pas à tout moment sentir qu'il est là, prêt à donner un coup de main, ce dialogue qui doit se suffire à lui-même et sur lequel tout repose, est plus ramassé, plus dense, plus tendu et survolté que le dialogue romanesque: il mobilise davantage toutes les forces du spectateur.“ (S. 1601) Vgl. auch: „Et surtout les acteurs sont là pour lui mâcher la besogne. Tout leur travail consiste justement à retrouver et à 
den Roman einzulösen: Der Leser der Romane Sarrautes soll in den Zustand einer angespannten Unruhe versetzt werden, die es ihm ermöglicht, sich jederzeit auf plötzliche „impulsions meutrières“ einzustellen:

Le dialogue, tout vibrant et gonflé par ces mouvements qui le propulsent et le sous-tendent, serait, quelle que soit sa banalité apparente, aussi révélateur que le dialogue de théâtre. (S. 1604)

Le lecteur, sans cesse tendu, aux aguets, comme s'il était à la place de celui à qui les paroles s'adressent, mobilise tous ses instincts de défense, tous ses dons d'intuition, sa mémoire, ses facultés de jugement et de raisonnement: un danger se dissimule dans ces phrases douceâtres, des impulsions meurtrières s'insinuent dans l'inquiétude affectueuse, une expression de tendresse distille tout à coup un subtil venin. (S. 1606)

Der Sarrautsche Dialogroman ist in hohem Maße sprachbewusst und versetzt den Leser in eine permanente Wachsamkeit gegenüber der Sprache. Er ist in der Kunst des Impliziten geschult, die die allerbanalsten Alltagskonversationen durchzieht. Der Roman versteht sich als Schule einer ,sous-conversation', in der Literatur und Realität angeblich nicht mehr zu unterscheiden sind:

Mais le lecteur n'est que rarement tenté de se départir de sa vigilance. Il sait qu'ici chaque mot compte. Les dictons, les citations, les métaphores, les expressions toutes faites ou pompeuses ou pédantes, les platitudes, les vulgarités, les maniérismes, les coq-à-l'âne qui parsèment habilement ces dialogues ne sont pas, comme dans les romans ordinaires, des signes distinctifs que l'auteur épingle sur les caractères des personnages pour les rendre mieux reconnaissables, plus familiers et plus „vivants“: ils sont ici, on le sent, ce qu'ils sont dans la réalité: la résultante de mouvements montés des profondeurs, nombreux, emmêlés, que celui qui les perçoit au-dehors embrasse en un éclair et qu'il n’a ni le temps ni le moyen de séparer et de nommer. (Ebd.)

\section{2 ,Tropismen ${ }^{6}$ in der indirekten Kommunikation}

In ihrem Erstlingswerk Tropismes ${ }^{9}$ werden solche Überlegungen durchgespielt. Exemplarisch lässt sich das an zwei Erzählstücken zeigen, dem zwölften Erzählstück über einen Professor des Collège de France und dem zweiten Erzählstück von jenem Mann, der die uferlosen Konversationen seiner Frau mit der Köchin der Familie als Bedrohung wahrnimmt.

reproduire en eux-mêmes, au prix de grands et longs efforts, les mouvements intérieurs infimes et compliqués qui ont propulsé le dialogue [...]”(S. 1602)

$9 \mathrm{Zu}$ den Tropismes vgl. grundsätzlich Jutta Lietz, „Zu Stil und Struktur der Tropismes von Nathalie Sarraute“, Romanistisches Jahrbuch Bd. 25/1974, S. 154-173. 


\section{XII}

Dans ses cours très suivis au Collège de France, il s'amusait de tout cela.

Il se plaisait à farfouiller, avec la dignité des gestes professionnels, d'une main implacable et experte, dans les dessous de Proust ou de Rimbaud, et étalant aux yeux de son public très attentif leurs prétendus miracles, leurs mystères, il expliquait „leur cas“.

Avec son petit œil perçant et malicieux, sa cravate toute faite et sa barbe carrée, il ressemblait énormément au Monsieur peint sur les réclames, qui recommande en souriant, le doigt levé: Saponite - la bonne lessive, ou bien la Salamandre modèle: économie, sécurité, confort. „Il n’y a rien“, disait-il, „,vous voyez, je suis allé regarder moi-même, car je n’aime pas m’en laisser accroire, rien que je n’aie moi-même mille fois déjà étudié cliniquement, catalogué et expliqué.

„Ils ne doivent pas vous démonter. Tenez, ils sont entre mes mains comme des petits enfants tremblants et nus, et je les tiens dans le creux de ma main devant vous comme si j'étais leur créateur, leur père, je les ai vidés pour vous de leur puissance et de leur mystère, j’ai traqué, harcelé ce qu'il y avait en eux de miraculeux.

„Maintenant, ils sont à peine différents de ces intelligents, de ces curieux et amusants loufoques qui viennent me raconter leurs interminables histoires pour que je m'occupe d'eux, les apprécie et les rassure. „Vous ne pouvez pas plus vous émouvoir que mes filles quand elles reçoivent leurs amies dans le salon de leur mère et bavardent gentiment et rient sans se soucier de ce que je dis à mes malades dans la pièce voisine“.

Ainsi il professait au Collège de France. Et partout alentour, dans les Facultés avoisinantes, aux cours de littérature, de droit, d'histoire ou de philosophie, à l'Institut et au Palais, dans les autobus, les métros, dans toutes les administrations, l'homme sensé, l'homme normal, l'homme actif, l'homme digne et sain, l'homme fort triomphait.

Evitant les boutiques pleines de jolis objets, les femmes qui trottinaient alertement, les garçons de café, les étudiants en médecine, les agents, les clercs de notaire, Rimbaud ou Proust, arrachés de la vie, rejetés hors de la vie et privés de soutien, devaient errer sans but le long des rues, ou somnoler, la tête tombant sur la poitrine, dans quelque square poussiéreux. ${ }^{10}$

Die Erzählung Nr. XII ist ein Beispiel einer vertikal ausgerichteten Kommunikation: Der Protagonist ist Professor am Collège de France und Arzt (,,je dis à mes malades“), offenkundig auf dem Fachgebiet der Psychologie bzw. Psychiatrie tätig"11. Seine Rolle in einer übergeordneten Position wird in ihrer Vertikalität mehrfach gesteigert: Er lehrt nicht an irgendeiner Institution, sondern an der angesehensten Bildungseinrichtung Frankreichs, an die seit dem 16. Jahrhundert die besten Köpfe berufen werden. Und eine weitere bei Sarraute überaus beliebte vertikale

10 Tropismes, in: Euvres complètes, S. 1-32, hier: S. 18 f.im Folgenden im laufenden Text nach dieser Ausgabe zitiert.

11 Vorbild für die Figur war vermutlich Pierre-Marie-Félix Janet, der 1902 bis 1934 den Lehrstuhl für experimentelle Psychologie am Collège de France innehatte und in seiner Dissertation L'Automatisme psychologique als einer der ersten über den Unterschied zwischen Unterbewusstsein und Bewusstsein gearbeitet hat. 
Figurenkonstellation, die von Vater und Kind, bekräftigt seine Stellung: Er sieht sich wie ein Vater, der die Autoren, die er untersucht, wie Kinder in seinen Händen hält.

Die Lehre an seiner ehrwürdigen Institution ist für den Professor ein höchst amüsantes Unterfangen: „il s’amusait de tout cela“. Seine tiefenpsychologischen Auslegungen literarischer Texte Rimbauds und Prousts sowie deren Übermittlung an seine Studenten sieht er als ein Spiel. Sinn des Spiels ist es, über eine direkte Kommunikation die ,sous-conversation' der beiden Autoren derart zu erhellen, dass sie sich sozusagen auflöst: „Il n’y a rien, disait-il“. Und die Studenten machen dieses Spiel offenbar mit, da sie seine Veranstaltungen zahlreich besuchen „(ses cours très suivis“) und mit Aufmerksamkeit („,son public très attentif“) verfolgen. Schaut man genauer auf die Tätigkeit des Professors, tun sich unter dieser Art der direkten Ansprache durchaus fragwürdige ,sous-conversations“ auf: Die Berufung des Professors („Ainsi il professait au Collège de France“) ist ein „farfouiller [...] dans les dessous de Proust et de Rimbaud“. Er behandelt die Literatur beider Autoren als „Fall“. Dieser wird klinischen Studien unterzogen; alles wird katalogisiert und erklärt. Der Professor sucht auf diese Weise die Texte zu entzaubern; er entledigt die Literatur beider Autoren jedweder Magie. Der literarische Schöpfungsprozess der beiden Schriftsteller wird geradezu klinisch seziert und seiner geheimnisvollen Eigenschaften entleert: „[...] je les ai vidés pour vous de leur puissance et de leur mystère [...]“. Proust und Rimbaud werden herabgestuft und in die Reihe bloß intelligenter Menschen eingerückt, deren „Verrücktheit“ („loufoques“) sich auf dem Niveau der Klientel des Professors bewegt: Ihre Geschichten haben den gleichen Stellenwert wie die seiner Patienten (,à peine différent de ces intelligents, de ces [...] loufoques qui viennent me raconter leurs interminables histoires“).

Der solcherart durch messerscharfe, scholastisch anmutende Analysen bewerkstelligte Abstieg der Literatur aus dem Reich einer schöpferischen Tätigkeit in den einer intelligenten Kunst im Sinne von bloßem ,Können` läuft parallel zum gleichzeitigen Aufstieg des Professors: In dem Maße, indem er durch seine Analysen den schöpferischen Prozess der Literatur zum Erliegen bringt, in dem Maße erhebt er sich selbst in die Rolle eines machtvollen Schöpfers: „[...] ils sont entre mes mains comme des enfants tremblants et nus, et je les tiens dans le creux de ma main devant vous comme si j'étais leur créateur“.

Der Tropismus liegt hier in der Groteske, die Abstieg und Aufstieg aufeinanderprallen lässt. Er entsteht aus den Widersprüchen, die sich auftun. So stellt sich wohl kaum jemand den Schöpfer vor: mit einer akkurat gebundenen Krawatte, einem quadratisch gestutzten ebenfalls akkuraten Bart und vor allem nicht mit einem „œil perçant et malicieux“. Der echte Schöpfer benötigt auch nicht professionelle Gesten, die ihm erst seine Würde verleihen („la dignitité des gestes profes- 
sionels"); die Hand des Schöpfers bedarf auch nicht adjektivischer Präzisierungen wie „implacable“ und „experte“. Die professionellen Gesten des Professors, d.h. seine Tricks, dienen der Authentifizierung seiner Analysen: Sie verdecken seine eigentliche Motivation. Unter dem Vorwand, die Studenten vor der Allgewalt der Autoren beschützen zu wollen, verbirgt sich ein grundlegender ,amourpropre' des Professors: Er will sich in seiner selbstverordneten Beschützerrolle bei den Studenten beliebt machen. Die Autoren werden von ihm demythifiziert, damit sie die Studenten nicht einschüchtern und erdrücken („Ils ne doivent pas vous démonter“). Er zeigt allen im Hörsaal - „devant vous“ -, wie sehr er beide Schriftsteller wie „zitternde und nackte Kinder“ in seiner Hand hält. Extra für die Studenten habe er die Autoren um ihre geheimnisvolle Macht gebracht, sie sozusagen „entleert“: „,...] je les ai vidé pour vous de leur puissance et de leur mystère[...]“.

Es ist diese Eitelkeit gepaart mit der Erscheinung als allmächtige Professorenfigur, die ihn zu einer grotesk-komischen Gestalt werden lässt und den Tropismus eines Unwohlseins erzeugt, von dem man sich nur durch Lachen befreien kann. In seiner Schrift Le Rire. Essai sur la signification du comique (1899) hatte Henri Bergson den Vorgang des Lachens als eine Befreiung in einer Situation beschrieben, die perfekt auf die geschilderte bei Sarraute passt:

Du mécanique plaqué sur du vivant, voilà encore notre point de départ. D’où venait ici le comique? De ce que le corps vivant se raidissait en machine. [...] Est comique tout incident qui appelle notre attention sur le physique d'une personne alors que le moral est en cause. ${ }^{12}$

Aus der schöpferischen Kraft der Literatur, der Vitalität ihrer Autoren, macht der so akkurat auftretende Professor des Collège de France einen pathologischen Fall, den einer klinischen Resektion und Leichenschau. Und im Zuge seiner hermeneutischen Offenbarungen werden nicht nur die Autoren zu gewöhnlichen Menschen des alltäglichen Lebens herabgewürdigt; auch der Professor sinkt zu einer leblosen Figur herab; er wird vom Erzähler letztlich in die Phalanx der durch und durch gewöhnlichen Menschen eingereiht. Dem Erzähler drängen sich Vergleiche auf, die durch ihren bathetischen Charakter Komik erzeugen. Die Haltung des Professors gegenüber der Literatur erinnert den Erzähler an die Ofenreklame der Firma „La Salamandre“ mit dem Slogan „économie, sécurité, comfort“, auf deren Werbeplakat seinerzeit ein gemütlich rauchender, vor einem Kaminofen sitzender Herr abgebildet war. Seine Lehre ,ex cathedra' mit dem durchdringenden und boshaften Blick erinnert ihn an den Mann mit erhobenem Zeigefinger aus der

12 Paris, Alcan, 1938, S. $51 \mathrm{f}$. 
Waschmittelreklame „Saponite, la bonne lessive“, geht doch der Professor auch keiner anderen Tätigkeit nach, als die Autoren Proust und Rimbaud von ihrer Aura reinzuwaschen.

Der Professor löst sich mit seiner analytischen Sezierung der literarischen Aura gleich selbst mit auf: Die Analyse der Texte führt ins Nichts. Die Hörer seiner Vorlesung sollen am Ende ihre Aufregung verlieren (,[...] vous ne pouvez plus vous émouvoir [...]“). Die Autoren sollen sie so wenig bewegen wie das Geschwätz der Töchter des Professors mit ihren Freundinnen im Nebenraum seines Behandlungszimmers, in dem weder den Geschichten seiner Patienten besondere Bedeutung zugemessen werden kann (,,interminables histoires“) noch seinen eigenen Worten an die Patienten. Der Professor, so heißt es in den beiden abschließenden Absätzen, versinkt mit seiner „profession“ letztlich in der Bedeutungslosigkeit alltäglicher Beschäftigungen, wie man sie in den anderen Fakultäten des Collège, am Institut (de France müsste man wohl ergänzen), im Gericht, im Autobus, der Metro sowie den Verwaltungen antrifft. Er versinkt in der Masse gewöhnlicher Menschen („l'homme sensé, l'homme normal, l'homme actif, l'homme digne et sain, l'homme fort"). Rimbaud und Proust, derart aus ihrem Leben gerissen, deren Vitalität sich vor allem aus ihrer, sous-conversation' speist, würden - so heißt es am Ende - mit gesenktem Kopf, wie schlafwandelnd im Menschenstrom der Boulevards verschwinden.

Sarrautes Erzählstück über das professorale, höchst amüsante Spiel der Welterklärung greift eine seit dem Mittelalter, spätestens seit der Gründung der europäischen Universitäten, gestellte Frage auf: Welchen Status hat ein Professor? Wie und was kann er lehren? Allein Gott kennt nach mittelalterlicher Vorstellung - um in der Metaphorik Sarrautes zu bleiben - die ,sous-conversations“ der Schöpfung. Allein er hat die Einsicht in die letzten Geheimnisse. Die mittelalterliche Universität bedarf, um die Zusammenhänge der Schöpfung zu beschreiben, der Kenntnis eines Grundlagenwissens, welches die ,artes“ vermitteln. Die ,Artes‘ sind einem bekannten Diktum der Antike zufolge, welches sich seit dem 12. Jahrhundert erneut großer Beliebtheit erfreut, ,simia naturae‘ bzw. im Mittelalter ,simia Dei ${ }^{{ }^{613}}$. Die freien Künste ahmen die Schöpfung nach; genauer genommen äffen sie sie nach. Dementsprechend wird der Professor der ,artes`immer wieder als ,Affe‘ beschrieben, der nachahmend die Natur und ihre Analogien erfassen und berechnen kann, nicht jedoch in der Lage ist, in die ,sous-conversation' der letzten Geheimnisse einzudringen. In den frühneuzeitlichen Enzyklopädien, insbesondere

13 Zur Wiederkehr des Topos im 12. Jahrhundert vgl. Ernst Robert Curtius, Europäische Literatur und lateinischen Mittelalter, Tübingen/Basel, Francke, ${ }^{11} 1993\left({ }^{1} 1948\right)$, S. 522 f. 
in Robert Fludds Utriusque Cosmi Historia (1617-1627) finden sich mehrere einschlägige ikonographische Darstellungen. Am Anfang des ersten Bandes wird in einer solchen Darstellung ,Sophia‘ als Verkörperung der göttlichen Weisheit ins Bild gesetz $\mathrm{t}^{14}$ :

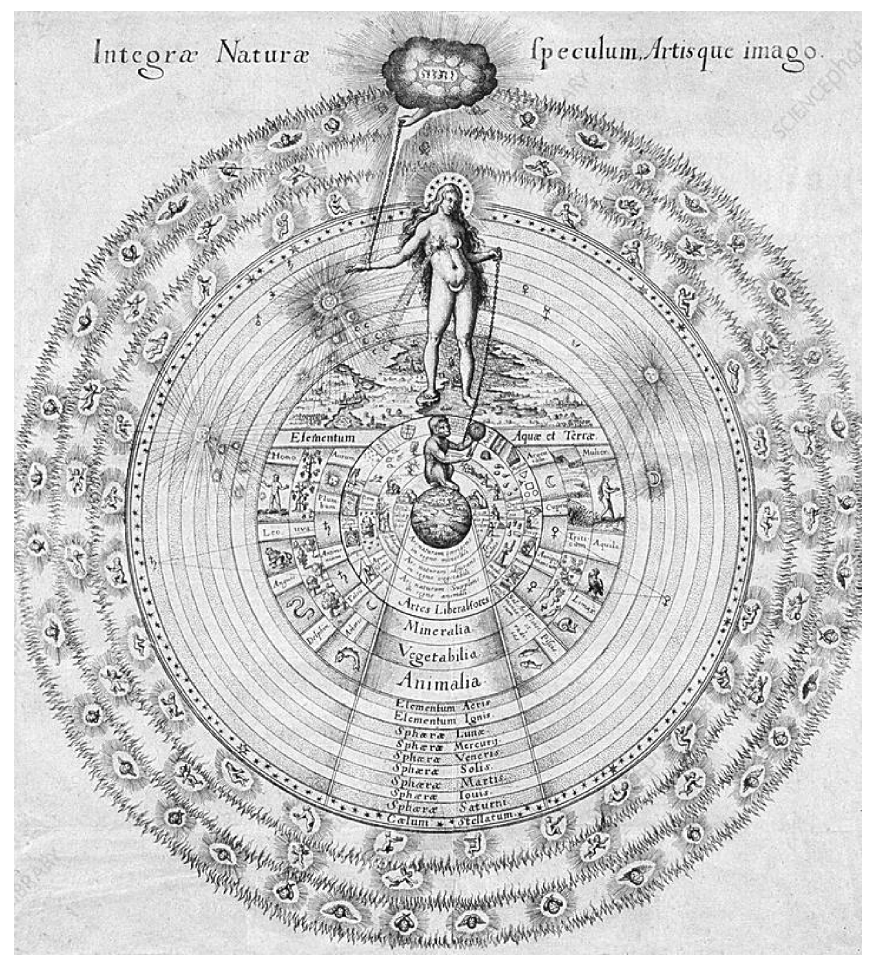

Sophia steht auf dem Rund der Natur, die ihrerseits deutlich von ihr getrennt unter der Herrschaft des Affen steht. Dieser hängt an der Kette der Weisheit. Der Affe sitzt mit einem Zirkel in der rechten Hand auf der Natur und vermisst einen künstlichen Globus, den er in der linken Hand hält und dessen Breiten- und Längengrade er berechnet. Seine höchste Leistung ist die Rechenkunst, was im Umkehrschluss bedeutet, dass ihm der Einblick in die Tiefendimensionen der Schöpfung vorbehalten bleibt. In einer weiteren Darstellung der Schrift ${ }^{15}$, im Traktat mit dem

14 Die Abbildungen finden sich in: Robert Flud, Utriusque cosmi maioris scilicet et minoris Metaphysica, physica atque technica Historia, 2 Bde., Oppenheim, Frankfurt 1617; online: http: //billheidrick.com/Orpd/RFludd/index.htm.

15 Die Abbildung findet sich ebenfalls online: http://billheidrick.com/Orpd/RFludd/index.htm. 
Titel De naturae simia seu Technica macrocosmi historia wird der Affe als schulmeisternder Professor mit Zeigestock verbildlicht, der die ,artes liberales ‘ und die ,artes technicae‘ lehrt. Der Professor (als Affe) gelangt somit nie über Erklärungen aus dem Bereich des menschlichen Könnens hinaus. Schöpferische Qualitäten gehören nicht zu seinem Reich.

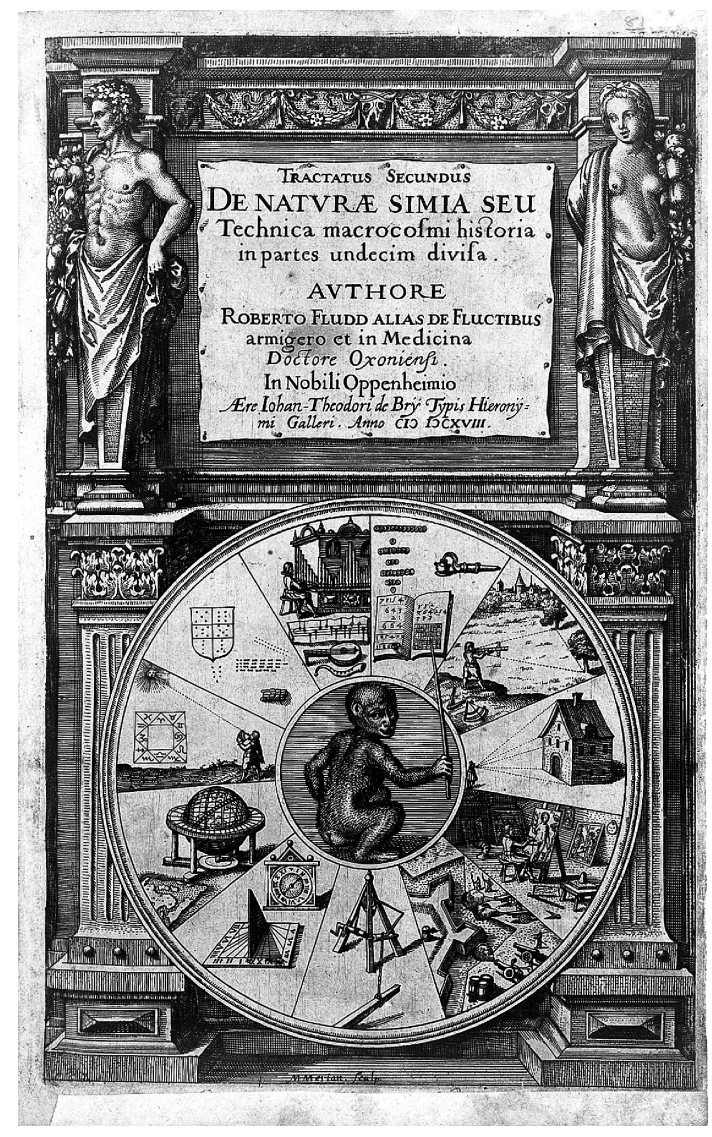

Jean de La Fontaine hat die Personifikation vom Affen als Professor aufgegriffen und sie in der Fabel Le Lion, le singe et les deux ânes (XI, 3) verarbeitet, die in wesentlichen Punkten Sarrautes Erzählstück tangiert. Der Löwe, Personifikation des absolutistischen Herrschers, fordert, um gut regieren zu können, dazu auf, ihn in die Grundsätze des menschlichen Umgangs, also die Moral, einzuweisen: „Le Lion, pour bien gouverner, / Voulant apprendre la morale [...]“(V.1f.). Dass seine Herrschaft offenbar nicht moralischen Ansprüchen genügt, ist eine Pointe gleich zu Beginn der Fabel. Herbeigeholt wird der Affe, seines Zeichens „maître ès arts“ 
(V. 3) und Professor (le Régent“, V. 516). Dieser liefert keine hehren Verhaltensgrundsätze der Moral, sondern empfiehlt dem Herrscher gemäß der negativ anthropologischen Tradition augustinischen Denkens das menschliche Grundverhalten schlechthin, den „amour-propre“, einzudämmen, um Lächerlichkeit und Ungerechtigkeit zu vermeiden. Dass der Professor auch gleich seine eigene „Profession“ als Musterfall der Eigenliebe enttarnt, ist offenbar bis zu Sarraute vorgedrungen:

Toute espèce, dit le Docteur,

(Et je commence par la nôtre)

Toute profession s'estime dans son cœur,

Traite les autres d'ignorantes,

Les qualifie impertinentes,

Et semblables discours qui ne nous coûtent rien.

(V. 22-27)

Und auch die professionellen körpersprachlichen Tricks des Professors bei Sarraute sind dem Affen bei La Fontaine bestens vertraut:

[...] ici-bas maint talent n'est que pure grimace,

Cabale, et certain art de se faire valoir,

Mieux su des ignorants que des gens de savoir.

(V. 32-34)

Mit dem Hinweis auf die „ignorants“ ist man dann bei jenen Durchschnittsmenschen angelangt, von denen Sarraute in den letzten beiden Abschnitten ihrer Erzählung berichtet und über deren Niveau auch der Affe bei La Fontaine mit der Erkenntnis nicht hinauskommt, nur aus Eigenliebe zu handeln. La Fontaines Fabel enthält eine weitere ,narratio zweier Esel, die sich über ihre Charakterisierung durch die Menschen beschweren. Der Fabeldichter setzt mit dieser Geschichte das Erasmische Sprichwort „asinus asinum fricat“ ins Bild ${ }^{17}$. Die Esel beschweren sich, dass die Menschen sie als „ignorant, d'esprit lourd, idiot“ (V. 42) ansehen und ihr Lachen von oben herab als ,iahen" bezeichnen. So lobt der eine Esel den andern und beide versichern sich auf diese Weise ihrer Außergewöhnlichkeit:

16 Im Dictionnaire françois : contenant les mots et les choses, plusieurs nouvelles remarques sur la langue françoise, ses expressions propres, figurées et burlesques, la prononciation des mots les plus difficiles, le genre des noms, le régime des verbes von César-Pierre Richelet heißt es unter dem Eintrag „Régent“: „Professeur qui enseigne une classe dans quelque Collège.“ (Genève, Widerhold, 1680, S. 281).

17 Erasmus von Rotterdam behandelt dieses Sprichwort in seiner Sammlung Adagia. 


\begin{abstract}
Ces Ânes, non contents de s'être ainsi grattés, S'en allèrent dans les cités

L'un l'autre se prôner: chacun d'eux croyait faire,

En prisant ses pareils, une fort bonne affaire,

Prétendant que l'honneur en reviendrait sur lui.

(V. 55-59)
\end{abstract}

Die augustinisch geprägte Erkenntnis, dass die Menschen nur ihrer Eitelkeit frönen und ihre Eigenliebe zum alleinigen Maßstab der Kommunikation machen, führt zur Lächerlichkeit, für die der Affe mit der Erzählung von den beiden Eseln ein Beispiel gibt. Den Affen treibt allerdings die gleiche Motivation wie die Esel, d. h. übertragen: Der Professor gehört zur Gruppe der gewöhnlichen Menschen, die an der Magie der Schöpfung keinen Anteil haben und somit nur ihrer Eigenliebe nachgehen können. Was diese Lektion für die Herrscher („Les puissances“, V. 61) bedeutet, an die sie bei La Fontaine gerichtet ist, überlässt der Dichter der Phantasie der Zuhörer.

Sarrautes Erzählungen, die sich nahezu alle mit der menschlichen Kommunikation befassen, fördern das zutage, was auf den ersten Blick im Umgang, der Konversation, verborgen bleibt, diese gleichwohl jedoch maßgeblich beeinflusst. Die menschliche Konversation ist für sie grundsätzlich agonal:

\footnotetext{
L'individu est le plus souvent en prise avec un autre qui cherche à le rabaisser et il déploie des stratégies pour empêcher son adversaire de le dévorer. Pour Sarraute, le monde humain est comme divisé en deux; celui des humiliés et celui des forts. ${ }^{18}$
}

Für den konkreten Fall des Professors am Collège de France gilt dies ganz besonders: Sein Auftritt ist, wie es zu Beginn heißt, ein Spiel. Die professorale Hermeneutik ist amüsant. Sie ist ein bloßes „Herumstöbern“ („farfouillement“). Der Professor ist so amüsiert wie der Herr aus der Seifenreklame (,[...] qui recommande en souriant [...]“). Er ist es schon allein deshalb, weil auch die von ihm analysierten Texte von Proust und Rimbaud seiner Meinung nicht besser sind als die langen Texte seiner ach so ,amüsanten abgefahrenen Patienten“ (,,amusants loufoques"). Diese behandelt er in seiner Praxis, begleitet von dem freundlichen Geschwätz und sorglosen Lachen der Töchter im Salon nebenan („[...] qui bavardent gentiment et rient sans se soucier [...]"). Dieses lustige Spiel ist jedoch ,unterschwellig - das macht den Tropismus und die irritierende Richtungsänderung des Erzählten aus - zugleich ein Spiel der Macht. Der Professor schwingt sich

18 Francine Cicurel, „L’avoué-inavoué à mi-voix : la confidence comme tropisme chez Nathalie Sarraute”, in: Cathérine Kerbrat-Orecchioni/Véronique Traverso (Hrsg.), Confidence/Dévoilement de soi dans l'interaction (Beiträge zur Dialogforschung. 37), Tübingen, Niemeyer, 2007, S. 139-152, hier: S. 143. 
mittels seiner hermeneutischen Künste auf Kosten der Autoren zum eigentlichen Schöpfer und Machthaber auf, obgleich er doch schon allein durch die akkurate äußere Erscheinung wie die Personifikation der Flaubertschen ,idée reçu' daherkommt. Und so geht folgerichtig im Lauf der Erzählung auch er zusammen mit der Erniedrigung der Autoren Proust und Rimbaud in die Reihe der „hommes sensés“ und „hommes normaux“ ein, die bezeichnenderweise erneut mit dem Machtmenschen endet („l'homme fort triomphant“). Sarrautes Tropisme XII ist eine Abrechnung mit der vertikalen Ausrichtung einer zentralistisch angelegten Situation, die dazu verführt, sich in der Kommunikation Machtpositionen anzumaßen. Aus der Sicht der von der Macht Betroffenen zeigt er die komisch-grotesken Dimensionen einer solchen Anmaßung auf.

Veranschaulicht der Tropisme XII die für Sarraute so wichtige Thematik unterschwelliger Macht und Gewalt, so befasst sich der Tropisme II mit eben diesem Thema in einer konkreten, ganz alltäglichen Kommunikationssituation und zeigt, aufgrund welcher Konstellationen und wie unmittelbar Irritationen durch bestimmte Formen der Machtausübung im Umgang der Personen ausgelöst werden. Die Protagonisten sind - für Sarraute typische - entpersönlichte Figuren: ein „il“, eine „elle“ und eine Köchin, die offenbar im Haushalt des Paars („,I““ und „elle“) beschäftigt ist:

Ils s’arrachaient à leurs armoires à glace où ils étaient en train de scruter leurs visages. Se soulevaient sur leurs lits: „C’est servi, c'est servi“, disait-elle. Elle rassemblait à table la famille, chacun caché dans son antre, solitaire, hargneux, épuisé. „Mais qu’ont-ils donc pour avoir l'air toujours vannés?“ disait-elle quand elle parlait à la cuisinière.

Elle parlait à la cuisinière pendant des heures, s'agitant autour de la table, s'agitant toujours, préparant des potions pour eux ou des plats, elle parlait, critiquant les gens qui venaient à la maison, les amis : „et les cheveux d'une telle qui vont foncer, ils seront comme ceux de sa mère, et droits; ils ont de la chance, ceux qui n'ont pas besoin de permanente“. -,Mademoiselle a de beaux cheveux“, disait la cuisinière, „ils sont épais, ils sont beaux malgré qu'ils ne bouclent pas“. - „Et un tel, je suis sûre qu'il ne vous a pas laissé quelque chose. Ils sont avares, avares tous, et ils ont de l'argent, ils ont de l'argent, c'est dégoûtant. Et ils se privent de tout. Moi, je ne comprends pas ça.“ - „Ah! non, disait la cuisinière, non, ils ne l'emporteront pas avec eux. Et leur fille, elle n'est toujours pas mariée, et elle n'est pas mal, elle a de beaux cheveux, un petit nez, de jolis pieds aussi.“- „Oui, beaux cheveux, c'est vrai, disait-elle, mais personne ne l'aime, vous savez, elle ne plaît pas. Ah! C'est drôle vraiment“.

Et il sentait filtrer de la cuisine la pensée humble et crasseuse, piétinante, piétinant toujours sur place, toujours sur place, tournant en rond, en rond, comme s'ils avaient le vertige mais ne pouvaient pas s'arreter, comme s'ils avaient mal au cœur mais ne pouvaient pas s'arreter, comme on se ronge les ongles, comme on arrache par morceaux sa peau quand on pèle, comme on se gratte quand on a de l'urticaire, comme on se retourne dans son lit pendant l'insomnie, pour se faire plaisir et pour se faire souffrir, à s'épuiser, à en avoir la respiration coupée... 


\begin{abstract}
„Mais peut-être que pour eux c'était autre chose.“ C'était ce qu'il pensait, écoutant, étendu sur son lit, pendant que comme une sorte de bave poisseuse leur pensée s'infiltrait en lui, se collait à lui, le tapissait intérieurement.

Il n’y avait rien à faire. Rien à faire. Se soustraire était impossible. Partout, sous des formes innombrables, „traîtres“( „c'est traître le soleil d'aujourd'hui, disait la concierge, c'est traitre et on risque d'attraper du mal. Ainsi, mon pauvre mari, pourtant il aimait se soigner..."), partout, sous les apparences de la vie elle-même, cela vous happait au passage, quand vous passiez en courant devant la loge de la concierge, quand vous répondiez au téléphone, déjeuniez en famille, invitiez des amis, adressiez la parole à qui que ce fût.

Il fallait leur répondre et les encourager avec douceur, et surtout, surtout ne pas leur faire sentir, ne pas leur faire sentir un seul instant qu'on se croyait différent. Se plier, se plier, s'effacer: „Oui, oui, oui, oui, c'est vrai, bien sûr“, voilà ce qu'il fallait leur dire, et les regarder avec sympathie, avec tendresse, sans quoi un déchirement, un arrachement, quelque chose d'inattendu, de violent allait se produire, quelque chose qui jamais ne s'était produit et qui serait effrayant.

Il lui semblait qu'alors, dans un déferlement subit d'action, de puissance, avec une force immense, il les secouerait comme de vieux chiffons sales, les tordrait, les déchirerait, les détruirait complètement.

Mais il savait aussi que c'était probablement une impression fausse. Avant qu'il ait le temps de se jeter sur eux - avec cet instinct sûr, cet instinct de défense, cette vitalité facile qui faisait leur force inquiétante, ils se retourneraient sur lui et, d'un coup, il ne savait comment, l'assommeraient.
\end{abstract}

Die Ausgangssituation beschreibt der einleitende Absatz, der gleich mehrere wichtige Informationen preisgibt: Ganz offenbar schaut das Paar (,ils“) unmittelbar nach dem Aufwachen in den Spiegel ihres Kleiderschranks. Die besondere Art des Schauens, des „scruter leurs visages“ verweist auf ein kritisch-skeptisches Mustern der Gesichter, auch des anderen. Das Schauen ist so intensiv, dass sie sich von dem Spiegel geradezu losreißen müssen. Wenn es dann im zweiten Satz heißt, dass jeder für sich isoliert und gereizt am Tisch Platz nimmt, ist der Spannungszustand der Familie umrissen. Eine besondere Rolle nimmt von vorneherein die Dame des Hauses („elle“) ein, die mit dem wiederholt vorgetragenen Ausruf „C'est servi“ die Familie an den Tisch treibt und die Köchin dann fragt, warum eigentlich alle so erschöpft aussähen.

Der Grund für die Gereitztheit tritt alsbald deutlicher zu Tage: Die Dame des Hauses redet ohne Unterlass. Sie spricht unentwegt schlecht über die Freunde, „les gens qui venaient à la maison, les amis“, während die Köchin stets antwortet und versucht, beschwichtigend die Spannung abzubauen. Im dritten Absatz wechselt die Perspektive zum Ehemann, der ausgestreckt auf seinem Bett die Reden aus der Küche hört. Hier wird die ganze Irration greifbar, vor allem hörbar: Das Gerede erscheint ihm als „pensée humble et crasseuse“. Die unermüdlichen Aktivitäten der Ehefrau und der Köchin scheinen sich im Kreise zu drehen. Die verwendeten Metaphern zeigen die vom Ehemann gefühlte Gewalt, die vom Ge- 
schehen in der Küche auf ihn ausgeübt wird: Die Worte „sickern durch“ und die schwindelerregenden Aktivitäten beider Damen werden assoziativ mit Vorstellungen körperlichen Unwohlseins oder Krankheiten belegt, von denen man sich Erleichterung zu verschaffen sucht. Während sich das Gerede wie „klebriger Geifer“ auf ihn legt und ihn innerlich verkleistert, denkt der Ehemann kurz darüber nach, ob man in der Küche die Dinge nicht ganz anders wahrnimmt.

Es folgt die fatalistische Erkenntnis, dass man sich dieser „bave poisseuse“ nirgendwo entziehen kann. Unter den „Erscheinungen des Lebens“ („les apparences de la vie") wird man überall und immer wieder solchen Irritationen ausgesetzt, bei der Begegnung mit der Hausmeisterin, am Telefon, beim Familienessen, bei der Begegnung mit Freunden oder wenn man an irgend jemanden das Wort richtet. Die einzig mögliche Reaktion scheint dem Ehemann die geschmeidige Unterwerfung zu sein, „se plier, s'effacer“. Der Gewalt des Tropismus kann man, so seine Einsicht, nur mit geheuchelter Sympathie und Zärtlichkeit entgegentreten, um den mit Sicherheit bevorstehenden Ausbruch zu vermeiden. Diese inoffensive Strategie kann aber auch - so vermutet der Ehemann nicht ohne gewisse Gewaltphantasien seinerseits - eine Strategie der totalen Vernichtung des Gegenübers sein. Sie würde die Frauen wie „schmutzige Lappen schütteln“ und sie letztlich vollständig zerstören (,les détruirait complètement“). Das Erzählstück endet mit seiner Überlegung, dass diese Strategie möglicherweise auf einem falschen Eindruck beruht und dass „elle“ und die Köchin ihm möglicherweise zuvorkommen könnten.

Das Erzählstück zeigt die unterschwellige Gewalt einer alltäglichen Kommunikation ${ }^{19}$. Sarrautes Texte greifen mündliche Kommunikationssituationen auf. Und so kommt es ihr - dies veranschaulicht insbesondere die räumliche Trennung der Kommunikationspartner - bei „il“" und „elle“ vor allem auf das Hören an. Genaues Zuhören ist eine Grundbedingung jedweder Konversation, um Tropismen erspüren und die Erkenntnis für Konversationsabläufe nutzen zu können. Das bevorzugte Stilmittel des Erzählstücks ist die Wiederholung, wie sie für mündliche Konversationen, insbesondere emotional gesteuerte Konversationen, typisch ist. „C'est servi, c'est servi“, sagt „elle“ gleich nach dem Erwachen. „Ils sont avares, avares tous, et ils ont de l'argent, ils ont de l'argent, c'est dégoûtant.”, schallt es dann später aus der Küche. Und ganz besonders die Wahrnehmung der immergleichen Bewegungen der Damen durch den Ehemann macht per Echoeffekt die Irritation auch für den Leser spürbar: „piétinante, piétinant toujours sur place, toujours sur place, tournant en rond, en rond, comme s'ils avaient le vertige“. Die

19 Zur „violence du texte“ bei Sarraute allgemein vgl. auch Georges Raillard, , Nathalie Sarraute et la violence du texte“, Littérature 1971, H. 2, S. 89-102. 
rhetorische Figur der Anapher bei den Vergleichen dieser Aktivitäten mit Reaktionen auf unangenehme bzw. krankheitsbedingte Körperbefindlichkeiten (,comme s’ils avaient“, „,comme s’ils avaient“, „comme on se ronge“, „,comme on arrache”, „comme on se gratte”, „comme on se retourne”) bringt letztlich beim Leser selbst das entsprechende Unwohlsein hervor. Die ,repetitio‘ erweckt zudem ein Gefühl der Fatalität („Il n’y a rien à faire. Rien à faire.“) angesichts der verräterischen Oberfläche der Worte, der Rede, die aufgrund der verdeckten Tropismen ,verräterisch“ ist („traîtres“ / „c'est traître [...]“ / „c'est traître [...]”). Die Gewalt der ,sous-conversation“ führt zu spürbaren Verhaltensgeboten, die der Ehemann sich auferlegt:

[...] surtout ne pas leur faire sentir, ne pas leur faire sentir un seul instant [...] Se plier, se plier, s'effacer: „Oui, oui, oui, oui, c’est vrai, bien sûr“, voilà ce qu'il fallait leur dire [...]

Sarrautes Anthropologie des Dialogs, in der Erkenntnisse aus der Hochphase der französischen Kommunikation im 17. Jahrhundert nachhallen ${ }^{20}$, in der die Moralistik die ,Kunst des Impliziten' bereits auf ihre negativ anthropologischen Bedingungen abgeklopft hatte, richtet sich auf die Erfahrbarkeit des Präsentischen $^{21}$. Die Autorin sucht nach einer neuen Erzählsprache, die gerade das Vorbzw. Nichtsprachliche unmittelbar spürbar machen will. Bei diesem Unterfangen ist die Nähe zum Theater unverkennbar. Es stellt sich die Frage, ob jene vorsprachlichen Erfahrungen, jene unwillkürlichen Erschütterungen, ausgelöst durch unvorhergesehene Ereignisse, durch bestimmte Gesprächssituationen oder Reaktionen, Worte, Reden, Gesten eines Gegenübers, nicht auch in einem Theaterdialog, der eine präsentische Kommunikation darstellt, zum Ausdruck gebracht werden können. Sarraute hat lange Zeit mit dem Theater als einem geeignetem Medium gehadert, um ihr dichterisches Anliegen zu übermitteln:

[...] il me semblait que le dialogue de théâtre était imcompatible avec ce que je cherchais à montrer [...] c'est-à-dire des mouvements intérieurs ténus, qui glissent très rapidement au seuil de notre conscience [...] Le dialogue seul, sans cette préparation que constitue un pré-

20 Inwieweit Sarrautes späterer autobiographischer Roman Enfance (1983) das dem autobiographischen Schreiben zugrundeliegende augustinische Paradigma der ,vocatio‘ zur ,conversio in litteris‘ verpflichtet ist und dieses transformiert, hat eindrucksvoll Maria Moog-Grünewald gezeigt („Konstellationen der Berufung. Nathalie Sarrautes Enfance in der Tradition Augustins“, in: Patricia Oster/Karlheinz Stierle (Hrsg.), Legenden der Berufung [Neues Forum für Allgemeine und Vergleichende Literaturwissenschaft. 49], Heidelberg, Winter, 2012, S. 47-59, bes. S. 55-59). 21 Zur Erfahrung des Präsentischen vor dem Hintergrund zeitgenössischer philosophischer Entwürfe vgl. Berit Callsen, „Präsenz. „...une substance coule, se répand...“ - Präsenzeffekte bei Nathalie Sarraute“, in: Antonius Weixler/Lukas Werner (Hrsg.), Zeiten erzählen. Ansätze Aspekte - Analysen (Narratologia), Berlin/Boston, De Gruyter, S. 431-447. 
dialogue, était pour moi impossible, impensable. [...] Le prédialogue, la sous-conversation, qui pousse le dialogue, le produit, montre au contraire au lecteur tout ce qu'il m'est possible de lui montrer. [...] Comment [...] écrire des pieces de théâtre où il n’y a rien d'autre que le dialogue? Il doit à lui seul faire sentir. Et quand à l'action extérieure, si importante au théâtre, elle est à peu près absente de mes livres. ${ }^{22}$

Als ihr dann jedoch durch den deutschen Kunsthistoriker Werner Spies angetragen wurde, Stücke für den Süddeutschen Rundfunk zu verfassen, hat Sarraute überlegt, wie der Theaterdialog auch für ihr Anliegen fruchtbar gemacht werden kann. Dialog und „pré-dialogue“ müssen zusammenfallen. Sarraute stellt sich der Herausforderung einer quasi erhöhten Präsenz, in gewöhnlichen Worten und in einer gewöhnlichen Sprache die sinnlichen, gefühlten Eindrücke unmittelbar und ohne Vorbereitung zu vermitteln:

Ce qui dans mes roman aurait constitué l'action dramatique de la sous-conversation, du prédialogue, où les sensations, les impressions, le „ressenti“ sont communiqués au lecteur à l'aide d'images et de rythmes, ici se déployait dans le dialogue lui-même. La sous-conversation devenait la conversation. [...] Il faut que la sensation, le ressenti, passe vite, ait une force d'impact immédiate, porté par des mots familiers. (S. 1708 f.)

In dem späten Theaterstück Pour un oui ou pour un non aus dem Jahr 1986, dem am häufigsten aufgeführten und sogar verfilmten Stück Sarrautes, tritt diese Konzeption mustergültig zu Tage. Der Protagonist H. 1 besucht H. 2, um ihn wegen dessen auffälliger Zurückhaltung in der letzten Zeit zur Rede zu stellen („,[...] je voudrais savoir... que s'est-il passée? Qu'est-ce que tu as contre moi ?“23). Innerhalb einer Stunde, in der sich die beiden unterhalten, treten die unterschiedlichsten Animositäten aufgrund der unterschiedlichsten Eindrücke zutage, wobei die Autorin offen lässt, ob es zum endgültigen Bruch zwischen beiden kommt. Im Medium der Mündlichkeit, dem Theater, geht es noch mehr als in dem der Schriftlichkeit, dem Roman und der Geschichte, darum, mit der Kunst des Impliziten zu arbeiten, die allerdings bei Sarraute eine mehr unbewusste als bewusste Weise der Erzeugung von Tropismen geworden ist. Verschiedene sprachliche Verfahren der Herstellung des Impliziten wie Unterstellungen, Andeutungen, Schweigen, Betonungen etc. bringen unterschiedliche Formen sprachlicher Gewalt zum Ausdruck. Nach der Eingangsaufforderung von H.1 an H.2 kommt es nach zahlreichen Ausweichmanövern schließlich zu einem Geständnis:

22 Le Gant retourné (1974), in: Euvres completes, S. 1707-1713, hier: S. 1707 f. Im Folgenden nach dieser Ausgabe im laufenden Text zitiert.

23 Pour un oui ou pour un non, in: Euvres completes, S. 1495-1515, hier: S. 1497. Im Folgenden nach dieser Ausgabe im laufenden Text zitiert. 
H. 2: Eh bien... tu m'as dit il y a quelque temps... tu m'as dit... quand je me suis vanté de je ne sais plus quoi... de je ne sais plus quel succès... oui... dérisoire... quand je t'en ai parlé... tu m’as dit: „C’est bien... ça...“

H. 1: Répète-le, je t'en prie... j’ai dû mal entendre.

H. 2, prenant courage: Tu m’as dit: „C’est bien... ça...“ Juste avec ce suspens... cet accent... [...]

H. 1: Et alors je t’aurais dit: „'’est bien, ça?“

H. 2, soupire: Pas tout à fait ainsi... il y avait entre „C'est bien“ et „ça“ un intervalle plus grand: „C’est biiien... ça...“ Un accent mis sur „bien“... un étirement : „biiien...“ et un suspens avant que „ça“ arrive... ce n’est pas sans importance. (S. 1499)

Anlass des Bruchs zwischen beiden (den H. 2. bestreitet) ist die seinerzeitige Aussprache der Formel durch H. 1: „C’est bien ça.“ H. 2 sieht in der Intonation der positiven Allerweltsformel, genauer der Dehnung des ,biiien“ und der längeren Sprechpause vor der Äußerung des ,ça', eine Form der Herablassung („condescendant“, S. 1501) Allein durch die Intonation ist das Gesagte von H. 2 als Angriff verstanden worden. Die lokutionäre Ebene des Gesagten steht im Widerspruch zur illokutionären; der Zweck der Sprachhandlung, hier eine als negativ verstandene Herabsetzung, widerspricht der Aussage in Form einer positiven, wohlwollend zustimmenden Bemerkung. Auf diese Weise erweist sich der Theaterdialog über das schiere ,Nichts‘ ganz plötzlich und unvermutet als Dialog über ein ,BeinaheNichts‘, dem eine Fülle vermuteter Intentionen entspringt und dem Gespräch neue unerwartete Wendungen verleiht.

Celui qui écoute perçoit certaines fréquences, qu'un autre - moins musicien, moins sensible peut-être - n'entendrait pas. Le ton, l'expression, la mimique et le choix des mots sont ce qui réellement constitue le flux secret de ce qui relie, éloigne, rapproche un être parlant d'un autre. ${ }^{24}$

Die hinter dieser Beweglichkeit des Gesprächs stehende Anthropologie konzipiert das Ich als eine täuschende Erscheinung, welches sich der inneren Regungen selbst kaum bewusst ist und im Verlauf einer Unterhaltung einerseits weitere unerwartete Regungen durchläuft und sich andererseits über seine Äußerungen verrät. Ein später Roman Sarrautes wird diesen Aspekt über die volle Länge der Erzählung ausspielen.

Während die meisten Erzählungen, Romane und Theaterstücke die zentrale Frage der Vertikalität der Kommunikation reflektieren, kommt im Roman Vous

24 Cicurel, „L’avoué-inavoué“, S. 150. Vgl. auch Béatrice Godart-Wendling/Layla Raïd, „Le clapet de la sourcière: les implicites de la violence verbale dans le théâtre de Sarraute”, in: Iuliana-Anca Mateiu (Hrsg.), La violence verbale: description, processus, effets discursifs et psycho-sociaux, Cluj-Napoca, Presa Universitara Clujeana, 2017, S. 195-210. 
les entendez? (1972) der für die französische Kommunikation so zentrale Aspekt der spielerischen Leichtigkeit des ,art de l'implicite' hinzu' ${ }^{25}$. In einem Haus auf dem Land besucht ein Freund den Hausherrn und nimmt eine Tierstatue vom Kamin, um sie betrachten zu können. Währenddessen verabschieden sich die Kinder des Hauses höflich von den beiden älteren Herren und ziehen sich lachend in die Schlafräume zurück. Angesichts dieser nichtigen, quasi inexistenten Handlung geht es darum, die Wirkungen dieses Lachens, der unbeschwerten ,légèreté‘ auf den Hausherrn zu erspüren. Der Roman beginnt in medias res mit der Unterhaltung zwischen dem Hausherrn und seinem Freund:

Soudain il s'interrompt, il lève la main, l'index dressé, il tend l'oreille... Vous les entendez?... Un attendrissement mélancolique amollit ses traits... Ils sont gais, hein? Ils s'amusent... Que voulez-vous, c'est de leur âge... Nous aussi, on avait de ces fous rires... il n’y avait pas moyen de s'arrêter... ${ }^{26}$

Die gesamte Handlung des Romans wird in dem einen Moment des Lachens verdichtet, der in der Folge einen weitgehend vom Hausherrn geführten inneren Monolog bzw. Dialog mit imaginären Stimmen zeitigt, um die möglichen Bedeutungen dieses an der Oberfläche eigentlich völlig unbedeutenden Lachens für sich zu erschließen:

Dès ce moment tout était là, ramassé dans cet instant... Mais quoi tout? Il ne s'est rien passé. (S. 741)

Das Lachen ist irritiertend. Es wirft die Frage ,Jugend vs. Alter`auf und löst gleich zu Beginn beim Vater eine melancholische Stimmung angesichts der verlorenen Leichtigkeit der Jugend aus (,des rires jeunes. Des rires frais. Des rires insouciants“, S. 737). Der Erzähler, der tiefe Einblicke in die Phantasien des Vaters entwickelt, tritt in einen Pseudodialog mit ihm ein: In der indirekten Form des konjunktivischen ,irrealis‘ fordert er den Vater zu der Gewalthandlung auf, alle Regeln des Anstands (,bienséance“) zu verletzen und das Lachen zu beenden:

Que le barbon irascible se lève brusquement sous les regards étonnés de l'ami en train de siroter paisiblement sa tasse de café, son verre de cassis, qu'il rompe brutalement toutes les

25 Zum speziellen Aspekt des Hörens in diesem Roman vgl. Laure Hesbois, „Vous les entendez? Nathalie Sarraute à l'écoute des signaux secrets. Tentative de décodage“, Atlantis. Critical Journal in Gender, Culture, and Social Justice Bd. 11/1986, H. 2, S. 88-96, sowie Noro Rakotobo d'Alberto, „Vous les entendez? L'écoute dans l'œuvre de Nathalie Sarraute”, Mnemosyne. La costruzione del senso Bd. 3/2010, S. 83-91.

26 Vous les entendez?, in: Euvres completes, S. 735-834, hier: S. 737. Im Folgenden nach dieser Ausgabe im laufenden Text zitiert. 
règles de la bienséance, qu'il monte l'escalier, frappe à la porte, l'ouvre furieusement, qu'il entre... Mais qu'est-ce que vous avez à rire comme ça? C'est insupportable, à la fin... et ils vont s'arrêter, se blottir dans les coins, tout effrayés, des nymphes effarouchées qu'un satyre surprend [...] (S. 741)

In der Perspektive der Kinder sähe dies anders aus, so der Erzähler. Eine solche Reaktion würde die Vorstellung des - freudianisch gesprochen - ständig präsenten Über-Ich aufrufen:

\footnotetext{
Mais il est toujours là à surveiller chaque geste, à réprimer le moindre élan, le plus léger signe d'insouciance, de liberté, toujours à scruter, à doser, à juger. N’a-t-on pas montré, comme il se devait, du respect? (Ebd.)
}

Das Lachen löst beim Vater Reflexionen über seine Rolle als Autoritätsperson aus. Hier sind erneut unterbewusst schwelende Machtfragen im Spiel: Dem „surveillant“ steht die kindliche Unbekümmertheit und Unschuld (,insouciance“, „innocent“, S. 741 f.) gegenüber. Es geht nicht nur um die Frage väterlicher Autorität. Auch seine Kompetenz und sein Sachverstand als Kunstkenner stehen auf dem Spiel:

Mais moi, voyez-vous... Moi... je dois dire que je n’ai jamais été un collectionneur... Jamais, n’est-ce pas? Ça ils le savent. Ça, vous le savez, là-bas? Je ne l'ai jamais été. Je n’en ai pas, vous me l'accorderez, le tempérament. Pas l'âme... Au contraire... Ça les fait sourire. Le contraire d'un collectionneur... (S. 744)

Der Roman dreht sich fortwährend um einen zentralen Mechanismus der indirekten Kommunikation: Wie kann die ,légèreté‘ zur Bedrohung der Autorität werden? $\mathrm{Zu}$ den Hochzeiten, in denen die französische Kommunikation in Europa Maßstäbe setzte, war das indirekte Sprechen ein Akt sozialer Anpassung, mit dem Ziel eine Konformität der Gesprächspartner herzustellen und füreinander Zuwendung aufzubringen. Der französische Charakter der Konversation bestand darin, so hatte es Mme de Staël formuliert, typische Sätze zu verwenden, die die Dinge aussprechen und dies zugleich auch nicht tun, um nicht allzu sehr Verbindlichkeiten einzugehen: „Il y a bien des phrases en effet en notre langue, pour dire en même temps et ne pas dire, pour faire espérer sans promettre, pour promettre même sans se lier. “27 Das Sprechen ist für Mme de Staël ein Konzert angenehmer Stimmen, bei dem die Worte nicht in erster Linie der Übermittlung von Gedanken und Gefühlen dienen. Sprechen ist ein Spiel, um die Geister zu animieren. Kommunikation ist oftmals nur ein Mittel, mit anderen zu interagieren, vor allem um

27 De l’Allemagne, S. 66 f. Vgl. auch oben, S. 77. 
seinen eigenen ,esprit‘ zur Schau zu stellen, an dem sich der Sprecher selbst wie elektrisiert berauscht:

Le genre de bien-être que fait éprouver une conversation ne consiste pas précisément dans le sujet de cette conversation [...] C'est une certaine manière d'agir les uns sur les autres, se faire plaisir réciproquement et avec rapidité, de parler aussitôt qu'on pense, de jouir à l'instant de soi-même, d'être applaudi sans travail, de manifester son esprit dans toutes les nuances par l'accent, le geste, le regard, enfin de produire à volonté une sorte d'électricité qui fait jaillir des étincelles $[\ldots]^{28}$

Dass diese ,grâce‘, die ,agréable légèreté‘, die ,variété d'esprit‘, wie es bei Mme de Staël immer wieder heißt, große Gefahren birgt, ist der Autorin bewusst:

C'est un excercice dangereux, mais piquant, dans lequel il faut se jouer de tous les sujets comme d'une balle lancée qui doit revenir à temps dans la main du joueur. ${ }^{29}$

Bei Nathalie Sarraute wird dieser Ball ganz anders zurückgespielt: Das Spiel ist bitterer Ernst. Der spielerische Charakter der indirekten Kommunikation wird als ,sous-conversation“ verstanden. Er verliert dadurch seine Funktion des Zusammenhalts der Gesprächspartner. In Vous les entendez? ist es gerade das spielerische Lachen der Kinder, welches die Irritation auslöst. Der Vater bezieht die ,légèreté‘ der Kinder fortwährend auf sich; er sieht darin einen Angriff auf seine Autorität und fragt sich, was er sich hat zu Schulden kommen lassen: „Mais peut-être sans le vouloir les a-t-il un peu brusqués?“ (S. 752) Die ,légèreté‘ setzt Ängste frei, die allein aufgrund ihres fluktuierenden Charakters nicht zu erklären sind:

Ce sont évidemment des questions auxquelles on ne doit pas se hâter de répondre. C'est grave d'enfermer dans des catégories rigides, d'étiqueter ce qui est encore fluctuant, changeant... Bien sûr, il y a toujours un espoir... [...] (S. 757)

Der Vater äußert einen geradezu konversationshistorischen Gedanken, wenn er im spielerischen Lachen der Kinder die Einstellung einer aristokratischen Verächtlichkeit sieht, welche ihm selbst vollends fehlt, und die sogleich bei ihm Angst-Komplexe auslöst:

[...] ils ont ce dédain aristocratique, cette indifférence qui leur donne une grâce, une élégance... qu'il n’a pas, ça lui manque à lui, un parvenu encore mal dégrossi [...] (S. 759)

28 S. 54 f. Vgl. dazu auch Wehinger, Conversation um 1800, S. 231.

29 S. 48. Vgl. Wehinger, Conversation um 1800, ebd. 
Sarraute wird auf diese Weise zur Erzählerin des Impliziten in der Kommunikation. Dies wird nicht länger als Kunst verstanden, sondern als Bedrohung ${ }^{30}$ :

La sous-conversation ainsi comprise est une pragmatique dépourvue de profondeur, une théâtralisation de l'implicite à l'œuvre dans toute interaction verbale. Cette théâtralisation fait toutefois abstraction de l'implicitation conversationnelle qui régit nos échanges: rien ne va plus de soi, le principe de coopération et la possibilité de s'entendre qui, malgré le cortège des malentendus et des incompréhensions, sous-tend la plupart de nos discussions, étant évacués: l'entente conversationnelle est rompue d'office. ${ }^{31}$

\section{Die französische Konversation hat bei Sarraute ihren Stellenwert einer positiven ,exception culturelle‘ verloren:}

La conversation représentée et instillée dans Vous les entendez? n'a rien à voir avec l'idéal d'un échange entre les savoirs et les gens du „monde“. Le cercle conversationnel se restreint à la société familiale et amicale ainsi qu'au lecteur, un lecteur privé impliqué à son tour dans la ronde des échanges familiers. ${ }^{32}$

In der ,Ära des Verdachts` arbeitet die Autorin eine Dimension der Konversation heraus, die laut Godo typisch für das gesamte 20. Jahrhundert ist:

La confiance mutuelle qui, dans la sociabilité classique, fait le fond de la conversation cède la place à une défiance généralisée. Le sujet parlant n'est plus maître de son langage: il n'est qu'une voix parmi d'autres, voix de tête, superficielle et trompeuse, étrangement émergée de la cacophonie enfouie au fond de son être. Le socle de certitude des idées claires qui permettaient que ce „qui se conçoit bien s'énonce clairement selon le précepte de Boileau“, a été remplacé par un sable mouvant - celui de l'inconscient et des ténèbres intérieures - qui rend toute parole précaire en faisant peser sur elle le soupçon de fallacité. Que disons-nous? Qui parle? qui? Que pouvons-nous connaître, inconnus à nous-mêmes, de ces étrangers qui nous environnent?33

30 Zur „Déconstruction du sujet“ in Vous les entendez? vgl. Sylvie Cadinot-Romerio, „Fiction et évélation: Vous les entendez? De Nathalie Sarraute “, Cahiers de narratologie Bd. 26/2014; online: https://journals.openedition.org/narratologie/6884, Abs. 31-38. Vgl. auch Fabien Demangeot, „La déconstruction de la notion de personnage dans l'œuvre de Nathalie Sarraute“, Studia Universitatis Petru Maia. Philologia Bd. 21/2016, S. 57-70.

31 Marie-Pascale Huglo, „Variations sur la conversation dans Vous les entendez? de Nathalie Sarraute“, Tangence Bd. 79/2005, S. 11-29, hier: S. 23.

32 Huglo, „Variations“, S. 28.

33 Godo, Une Histoire, S. 323. 
